\title{
Electrodermal Activity and Chronotropism for Transient Occlusion of Major Upper Arm Artery: A Quantitative Assessment of Autonomic Response
}

\author{
Ojashwi Nepal, ${ }^{1}$ Abhishek Ranjan Ray, ${ }^{2}$ Sony Pokhrel, ${ }^{2}$ Reena Kumari Jha' \\ 'Department of Physiology, Pre-Clinical Science Complex, Kathmandu University School of Medical Sciences, Chaukot, \\ Kavrepalanchok, Nepal, ${ }^{2}$ School of Science Kathmandu University, Dhulikhel, Nepal.
}

\section{ABSTRACT}

\section{Introduction}

Blood vessels in body respond to sympathetic upsurge and, occlusion of blood vessels supplying vital organs excites autonomic reactions, building up chaos in homeostasis. Autonomic response to occlusion of major artery, not supplying to vital organs, remains least explored in the medical literature. Thus, we have observed, in vivo, undulating autonomic response to heart beat and galvanic skin response for transient occlusion of left brachial artery, in this study.

\section{Methods}

Occlusion of brachial artery was maintained approximately $10 \mathrm{mmHg}$ above the systolic pressure by mercury manometer with cuff inflated at two inches above the cubital fossa of left hand. Heart rate and skin conductance was picked up by the transducers placed at appropriate locations on body surface in 40 subjects.

\section{Results}

Paired $t$ test, repeated measures ANOVA and Pearson correlation are utilized to compare the recorded variables. Heart rate is significantly decreased $(\mathrm{p}=0.000)$ and amplitude of galvanic skin response is significantly increased $(\mathrm{p}=0.000)$ in all subjects for induced occlusion of brachial artery in left arm. ANOVA compared among levels of pre-occlusion, occlusion and post-occlusion is significant for heart rate $(\mathrm{p}=0.000)$. Pearson correlation between GSR and heart rate is weakly inverse $(r=-0.028)$.

\section{Conclusions}

Episodic occlusion of artery does excite the autonomic reactions in healthy young adults. Simultaneous rise in parasympathetic and sympathetic response is an obligatory autonomic response for the brief occlusion of left brachial artery.

Keywords: brachial artery; chronotropic; galvanic skin response; occlusion.

Correspondance: Dr. Ojashwi Nepal, Department of Physiology, Pre-Clinical Science Complex, Kathmandu University School of Medical Sciences, Chaukot, Kavre, Nepal. Email: Ojashwi@kusms.edu.np. Phone: +977-9851087866. 


\section{INTRODUCTION}

Blood vessels in the body are innervated by sympathetic nerves and the elevated muscle sympathetic activity contributes to stiffening of the arteries. ${ }^{1}$ Ischemia to vital organs such as heart activates increased and sustained sympathetic activity. ${ }^{2}$ Myocardial infarction can have sustained sympathetic response for longer duration even after clinical course of treatment. The autonomic influence deriving the bodily reactions to the induced occlusion of large artery is a scarce in literature. And, information regarding application of skin conductance for functional physiological changes of autonomic output is limited. We wanted to study the autonomic response for innocuous occlusion of major artery in core and body surface and, correlate the reaction brought forth by the induced event. Thus, we have observed in vivo undulating autonomic response for change in heart beat and sympathetic skin response for transient occlusion of brachial artery in this study.

\section{METHODS}

The study was performed in convenience sampling of 40 subjects. Students from various faculties of university participated. The age of subjects ranged from 18-24 years and we included 40 subjects. Those who matched inclusion criteria were explained to the procedure and, were asked to visit physiology lab at medical school within the defined schedule, post lunch, in a day. Those who consented were continued with the procedure. Subjects rested for 10 minutes and the procedure was carried out which was designed to last for six minutes, splitted as, pre-occlusion, occlusion and post occlusion stages, each of two minutes. Occlusion of brachial artery was maintained approximately to $10 \mathrm{mmHg}$ above the systolic pressure of each subject by mercury manometer with cuff inflated at two inches above the cubital fossa of left hand. Skin conductance was measured by two electrodes strapped at distal phalanges of middle and ring fingers, in right hand in all subjects. Recordings obtained after the subjects were comfortable was stored for analysis. Deviation in pressure while maintaining occlusion was $\pm 6-8 \mathrm{mmHg}$ out of $10 \mathrm{mmHg}$ above the cut off value of systolic pressure in the subject. Heart Rate and skin conductance were measured as per defined protocol in the lab. Heart rate was picked up by the transducer placed at appropriate locations on body surface. Amplitude (Apeak) in micro Siemens and heart rate (HR) in beats per minute were observed from the table sheet generated by the professional version 7 software of the AD Instrument employed in the lab for the test. Average heart rate obtained were observed for chronotropicity. Skin conductance was measured throughout and, average amplitude of three different stages were recorded. Skin conductance and galvanic skin response (GSR) refers to the same very parameter i.e. amplitude (Apeak) recorded in subjects and, has been used interchangeably, without ambiguity, in this study.

\section{RESULTS}

Mean and standard deviation for amplitude values of skin conductance along with heart beats were computed utilizing software version 24 of SPSS. Paired $t$ test, repeated measures ANOVA and Pearson correlation are utilized to compare the recorded variables. Mean values of amplitude (Apeak) recorded for galvanic skin response (GSR) and heart rate (HR) are mentioned for genders in three different levels of pre-occlusion, occlusion and post-occlusion of brachial artery as given below in table 1 .

Table 1. Descriptive statistics with mean and standard deviation for GSR and heart rate.

\begin{tabular}{|l|l|l|l|l|l|}
\hline Parameter & Subjects (N) & Minimum value & Maximum value & Mean & Std. Deviation \\
\hline GSR preocclusion & 40 & -2.14 & 3.54 & 1.29 & 1.23 \\
\hline
\end{tabular}


Nepal et al. Electrodermal activity and chronotropism for transient occlusion of major upper arm..

\begin{tabular}{|l|l|l|l|l|l|}
\hline GSR occlusion & 40 & -1.34 & 5.65 & 2.77 & 1.58 \\
\hline GSR postocclusion & 40 & -1.24 & 4.58 & 2.05 & 1.30 \\
\hline HR preocclusion & 40 & 87.58 & 113.50 & 105.10 & 6.37 \\
\hline HR occlusion & 40 & 60.41 & 110.60 & 96.66 & 11.8 \\
\hline HR postocclusion & 40 & 90.29 & 113.80 & 104.76 & 6.30 \\
\hline FGSR preocclusion & 20 & -2.14 & 3.54 & .85 & 1.23 \\
\hline FGSR occlusion & 20 & .58 & 5.10 & 2.57 & 1.12 \\
\hline FGSR postocclusion & 20 & -.48 & 4.58 & 2.04 & 1.31 \\
\hline FHR preocclusion & 20 & 87.58 & 113.50 & 105.22 & 7.18 \\
\hline FHR occlusion & 20 & 77.85 & 110.60 & 98.23 & 9.45 \\
\hline FHR postocclusion & 20 & 90.29 & 112.90 & 104.87 & 7.29 \\
\hline MGSR preocclusion & 20 & -.64 & 3.14 & 1.74 & 1.09 \\
\hline MGSR occlusion & 20 & -1.34 & 5.65 & 2.98 & 1.94 \\
\hline MGSR postocclusion & 20 & -1.24 & 3.91 & 2.07 & 1.33 \\
\hline MHR preocclusion & 20 & 92.70 & 113.20 & 104.99 & 5.64 \\
\hline MHR occlusion & 20 & 60.41 & 109.50 & 95.10 & 13.85 \\
\hline MHR postocclusion & 20 & 95.90 & 113.80 & 104.66 & 5.32 \\
\hline
\end{tabular}

GSR; galvanic skin response, HR; heart rate, FGSR; female galvanic skin response, FHR; female heart rate, MGSR; male galvanic skin response, MHR; male heart rate

As depicted in table 2, skin conductance is increased and heart rate is decreased,
Repeated measures ANOVA was employed to compare three levels of procedure splitted

Table 2. Amplitude of galvanic skin response and heart rate compared between pre-occlusion versus occlusion.

\begin{tabular}{|c|c|c|c|c|}
\hline Variables & $\begin{array}{c}\text { Pre-occlusion } \\
\text { (Mean } \pm \text { SD) }\end{array}$ & $\begin{array}{c}\text { Occlusion } \\
\text { (Mean } \pm \text { SD) }\end{array}$ & Subjects & $P<0.05$ \\
\hline GSR & $1.29 \pm 1.23$ & $2.77 \pm 1.58$ & 40 & $0.000^{*}$ \\
\hline HR & $105.10 \pm 6.37$ & $96.66 \pm 11.81$ & 40 & $0.000^{*}$ \\
\hline
\end{tabular}

Heart rate; beats per minute, GSR; amplitude in micro Siemens

significantly $(\mathrm{p}=0.000)$, during occlusion of brachial artery. Paired ' $t$ ' test for amplitude values from GSR and beats of heart rate was performed in subjects to compare 'mean values' of pre-occlusion to occlusive state of brachial artery.

GSR; amplitude of galvanic skin response, HR; heart rate in pre, during and post occlusion of brachial artery. Heart rate compared in three levels showed significant difference $(p=0.000)$ when computed in all 40 subjects. Likewise, 20 male had significant difference $(p=0.000)$ in variation in heart beat compared in three levels as shown in table 3. 


\begin{tabular}{|c|c|c|c|c|c|}
\hline \multicolumn{6}{|l}{ Table 3. Repeated measures ANOVA compared } \\
among levels of pre-occlusion, occlusion and post- \\
occlusion for GSR and heart rate. \\
\hline Variables & $\begin{array}{c}\text { Subjects } \\
\text { (N) }\end{array}$ & Gender & $\begin{array}{c}\text { Approx. } \\
\text { Chi- } \\
\text { Square }\end{array}$ & df & 'p' value \\
\hline GSR & 40 & Both & 0.216 & 2 & 0.898 \\
\hline HR & 40 & Both & 26.512 & 2 & $0.00 *^{*}$ \\
\hline GSR & 20 & Female & 0.443 & 2 & 0.801 \\
\hline HR & 20 & Female & 3.605 & 2 & 0.165 \\
\hline GSR & 20 & Male & 0.120 & 2 & 0.942 \\
\hline HR & 20 & Male & 20.038 & 2 & $0.00 *^{*}$ \\
\hline
\end{tabular}

In paired samples ' $t$ ' test compared between male and female, computed separately for GSR and heart rate at three different levels of pre-occlusion, occlusion and post-occlusion, amplitude of GSR was significantly different $(p=0.033)$ at pre-occlusion, as shown below in table 4 .

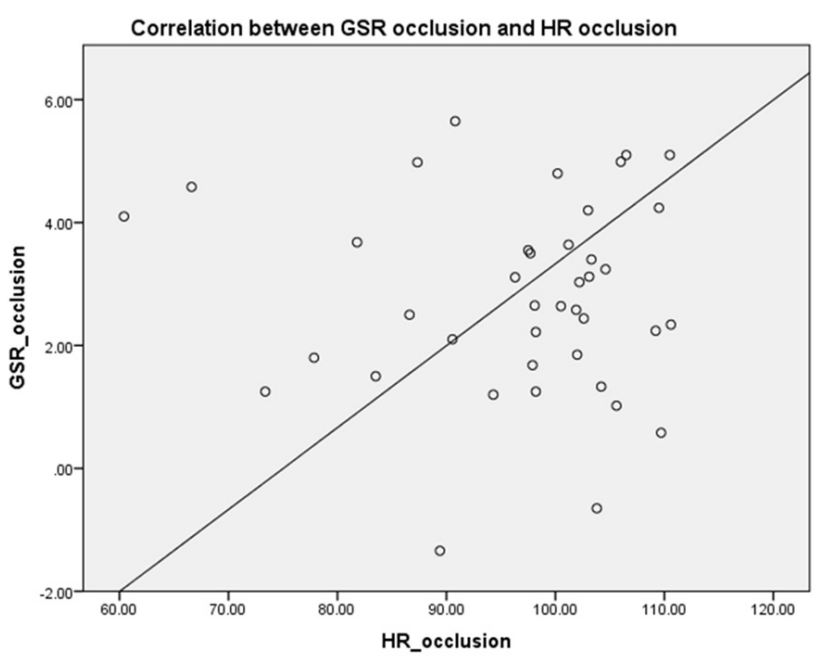

GSR measuredas amplitude in micro Siemens, HR in beats per minute

Figure 1. Pearson correlation between GSR and heart rate for induced occlusion of brachial artery in upper arm.

\section{DISCUSSION}

We wanted to observe the core and superficial autonomic response for brief occlusion of brachial artery through quantitative assessment of chronotropicity and electrodermal response.

Table 4. Variables compared between genders at levels of pre-occlusion, occlusion and post-occlusion.

\begin{tabular}{|c|c|c|c|c|c|}
\hline Levels & Variables & $\begin{array}{c}\text { Subjects } \\
\text { (N) }\end{array}$ & Male (Mean \pm SD) & Female (Mean $\pm S D)$ & $p<0.05$ \\
\hline \multirow[t]{2}{*}{ Pre-occlusion } & GSR & 20 & $1.74 \pm 1.09$ & $0.85 \pm 1.23$ & $0.033^{*}$ \\
\hline & $\mathrm{HR}$ & 20 & $104.99 \pm 5.64$ & $105.22 \pm 7.18$ & 0.909 \\
\hline \multirow[t]{2}{*}{ Occlusion } & GSR & 20 & $2.98 \pm 1.94$ & $2.57 \pm 1.12$ & 0.396 \\
\hline & $\mathrm{HR}$ & 20 & $95.10 \pm 13.85$ & $98.23 \pm 9.45$ & 0.457 \\
\hline \multirow[t]{2}{*}{ Post-occlusion } & GSR & 20 & $2.07 \pm 1.33$ & $2.04 \pm 1.31$ & 0.924 \\
\hline & $\mathrm{HR}$ & 20 & $104.66 \pm 5.32$ & $104.87 \pm 7.29$ & 0.916 \\
\hline
\end{tabular}

Correlation between heart rate and amplitude of conductance during occlusion of brachial artery is weakly inverse with $(\mathrm{r}=-0.028)$ as depicted in figure 1. Two compared variables are poorly correlated as the value $(r=-0.028)$ is quite close to zero. There is increment in amplitude of GSR with small fall in HR during induced occlusion of brachial artery.
Transiently induced occlusion of brachial artery in upper arm reduced heart beat and resulted in higher conductance over skin surface in our study. Event related galvanic skin response reflects the sympathetic activity and, heart rate drop denotes the parasympathetic overdrive.

It is known fact, sympathetic discharge upsurge 
in ischemic episodes but, clinical relevance of such response is often overseen and ignored, for the relevance of those information to immediate treatment is unavailing. Nevertheless, atypical presentations in clinics demand close and detail observation of underlying body response. Such presentation of the acute brachial artery occlusion with non-ST segment elevation myocardial ischemia (NSTEMI) in elderly patient has been reported in literature. ${ }^{3}$ Presentation of brachial artery occlusion has been attributed to the result of embolism, thrombosis due to trauma, arteriosclerosis and brachial artery catheterization. ${ }^{4,5}$ Since the presentation in clinic of such patients would vary from neurological, vascular, dermatological to musculoskeletal manifestations, the underlying physiological autonomic response though, clinically trivial for immediate treatment, is an important information to evaluate for chronic effects in health of patients.

In this light, here, we have compared skin conductance and heart rate for pre-occlusive state versus occlusive state in healthy young adults, shows a significant increment in autonomic outflow for the artificial occlusion of brachial artery. Mean and standard deviation are calculated for values obtained for preocclusion, during occlusion and post-occlusion of brachial artery in subjects as shown in table 1 of descriptive statistics. It also depicts maximum and minimum average values of skin conductance and heart rate.

As depicted in table 2, there is significantly $(p=0.000)$ higher conductance in skin brought about by heightened sympatho-cholinergic effluence to eccrine glands during brachial artery occlusion. On the contrary, heart rate is significantly $(p=0.000)$ reduced during occlusion of artery representing, the simultaneous heightened effluence of parasympathetic activity to the heart.

Literatures regarding use of skin conductance during event related perturbation in autonomic activity for arousal response, pain perception and emotional flare is numerous. And, has been coupled to electrocardiography and electroencephalography as an aid for diagnosis and detection of psychological and physiological responses..$^{6-8}$ It is well known that induced occlusion of large size forearm vessels can be utilized to assess the endothelial function which is relevant for assessment of cardiovascular health status. ${ }^{9,10}$ But, altered response of autonomic neural activity to occlusion of blood flow through brachial artery is observed, here. We did not observe the state of vasomotion through galvanic skin response, if at all, is brought about by large vessel obstruction. This was avoided and limited to autonomic response observation by occluding the vessel in the opposite limb to that of conductance measurement. Amplitude of skin conductance is 'Apeak' measured as GSR value that is an indicator of event related superficial sympathetic outflow.

Increased arterial distensibility in radial denervation has been discussed in literature in the light of stiffening effect of sympathetic nerve activity in arterial wall. ${ }^{11,12}$ Degree of sympathetic burst detected through skin conductance may assist to evaluate stiffness of artery.

Repeated measures ANOVA in table 3 for three different levels of recordings is significantly different for heart rate. Compared among males for heart rate changes in pre-occlusion versus 
occlusion to post-occlusion is significantly different $(p=0.000)$ and, the skin conductance has no significant variations. Fall in heart beat is significant that indicates parasympathetic overdrive.

Both variables were compared between genders in three different levels separately in table 4 that shows significant conductance difference with higher amplitude during preocclusion recording in male compared to female. There occurs no significant difference between genders for all other levels for heart rate and skin conductance but the response of two parameters are in opposite direction. Fall in heart rate during occlusion of brachial artery is pronounced in male. In our previous study, skin conductance level is greater in men than in females, consistent to recent finding for pre-occlusive observation and, amplification in galvanic response was greater during standing from sitting posture, without change in heart rate. ${ }^{13}$ In this study, perturbation in heart rate without postural change is significant, for occlusion of brachial artery.

During post occlusive recording heart rate rebounds but skin conductance decreases towards value of pre-occlusive state. During occlusion of brachial artery, the parasympathetic dominance to heart reduced beats but sympathetic drive caused rise in conductance over skin surface. Thereby, correlation between these parameters were computed during the arterial occlusion in figure 1 and, r=-0.028 was found with weak negative correlation. Skin conductance and heart rate are inversely correlated. This shows there is simultaneous overdrive of sympathetic and parasympathetic system for the maneuver with, higher superficial sympathetic outflow to eccrine glands and raised parasympathetic drive to the core in the heart during, induced arterial occlusion in healthy young adults. Immediately, after the occlusion is removed, blood flows through the vessel, the superficial sympathetic rise to eccrine begins to level off but, does not drop to the value of pre-occlusion state in two minutes. Heart rate, on the other hand, shoots up to the pre-occlusive state within the duration of two minutes. The drop of parasympathetic drive is prompt at the core and, levelling off of sympathetic outflow to body surface is gradual and sluggish.

We have observed, perturbation for induced transient occlusion to the left brachial artery in upper limb is detectable through significant drop in heart rate and, increment in skin conductance. Fall in parasympathetic overdrive is swift, at core, as demonstrated by sudden rise in heartbeat immediate, after the removal of occlusion. Sympathetic outflow to body surface is significant during arterial occlusion yet is moderate and steady, in healthy young adults. Further comparative study in different age groups of healthy adults versus patients of cardiovascular disease would unfold pathophysiologic evidence to bridge the gap, in understanding of underlying autonomic changes, in varied clinical states.

\section{CONCLUSIONS}

Autonomic perturbations, at core, for the vascular occlusive episode is obligatory in healthy state. Sympathetic ascendancy and parasympathetic amplification is simultaneous for the left upper arm artery occlusion.

\section{ACKNOWLEDGEMENTS}

We thank Ms. Kalpana Sharma at Department of Anatomy for timely help in statistical analysis during lockdown period of COVID-19. 


\section{REFERENCES}

1. Holwerda SW, Luehrs RE, Dubose L, Collins MT, Wooldridge NA, Stroud AK, et al. Elevated muscle sympathetic nerve activity contributes to central artery stiffness in young and middle-age/older adults. Hypertension. 2019;73(5):1025-35.

2. Jardine DL, Charles CJ, Ashton RK, Bennett SI, Whitehead M, Frampton $\mathrm{CM}$, et al. Increased cardiac sympathetic nerve activity following acute myocardial infarction in a sheep model. J Physiol. 2005;565(1):325-33.

3. Ologun GO, Bohan C, Lau T, Sultany M, Trecartin A, Wolfe Z, et al. Acute Brachial Artery Occlusion in an Elderly Patient With Acute Myocardial Ischemia. Cureus. 2017;9(9).

4. Hynes KM, Gau GT, Rutherford BD, Kazmier FJ, Frye RL. Effect of aspirin on brachial artery occlusion following brachial arteriotomy for coronary arteriography. Circulation. 1973;47(3):554-7.

5. Nijhuis HHAM, Müller-Wiefel $H$. Occlusion of the brachial artery by thrombus dislodged from a traumatic aneurysm of the anterior humeral circumflex artery. J Vasc Surg. 1991;13(3):408-11.

6. Setyohadi DB, Kusrohmaniah $S$, Gunawan SB, Pranowo, Prabuwono AS. Galvanic skin response data classification for emotion detection. Int J Electr Comput Eng. 2018;8(5):4004-14.

7. Goshvarpour A, Abbasi A, Goshvarpour A.An accurateemotion recognition system using ECG and GSR signals and matching pursuit method. Biomed J [Internet]. 2017;40(6):355-68. Available from: https:// doi.org/10.1016/j.bj.2017.11.001

8. Najafpour E, Asl-Aminabadi N, Nuroloyuni S, Jamali Z, Shirazi S. Can galvanic skin conductance be used as an objective indicator of children's anxiety in the dental setting? J Clin Exp Dent. 2017;9(3):e377-83.

9. Pyke KE, Dwyer EM, Tschakovsky ME. Impact of controlling shear rate on flowmediated dilation responses in the brachial artery of humans. J Appl Physiol. 2004 Aug;97(2):499-508.

10. Maltz JS, Tison GH, Alley HF, Budinger $\mathrm{TF}$, Owens CD, Olgin J. Measurement of brachial artery endothelial function using a standard blood pressure cuff. Physiol Meas. 2015;36(11):2247-2268. doi:10.1088/0967$3334 / 36 / 11 / 2247$

11. Giannattasio C, Failla M, Lucchina S, Zazzeron C, Scotti V, Capra A, et al. Arterial stiffening influence of sympathetic nerve activity: Evidence from hand transplantation in humans. Hypertension. 2005;45(4):608-11.

12. Giannattasio C, Vincenti A, Failla M, Capra A, Cirò A, De Ceglia S, et al. Effects of heart rate changes on arterial distensibility in humans. Hypertension. 2003;42(3):253-6.

13. Nepal O, Manandhar L, Rk J. Skin Conductance and RR Interval for Regulated Discrete Physiological Stimuli: A Two Prong Strategy to Detect Sympathetic Activation.Kathmandu Univ Med J. 2019;68(4):267-72.

Citation: Nepal O, Ray AR, Pokhrel S, Jha RK. Electrodermal Activity and Chronotropism for Transient Occlusion of Major Upper Arm Artery: A Quantitative Assessment of Autonomic Response. JCMS Nepal. 2020; 16(4);188-94. 